\title{
Proton Radioactivity from Highly Deformed Nuclei
}

A.A. Sonzogni ${ }^{1}$, C.N. Davids ${ }^{1}$, P.J. Woods ${ }^{2}$, D. Seweryniak ${ }^{1,3}$, J.C. Batchelder ${ }^{4}$, C.R. Bingham ${ }^{5}$, T. Davinson ${ }^{2}$, D.J. Henderson ${ }^{1}$, R.J. Irvine ${ }^{2}$, G.L. Poli ${ }^{6}$, J. Uusitalo $^{1}$ and W.B. Walters ${ }^{3}$.

${ }^{1}$ Argonne National Laboratory, 9700 South Cass Avenue, Illinois 60439 ${ }^{2}$ University of Edinburgh, Edinburgh EH9 3JZ, United Kingdom

${ }^{3}$ Department of Chemistry, University of Maryland, College Park, MD 20742

${ }^{4}$ UNIRIB, Oak Ridge Associated Universities, Oak Ridge, TN 37381

${ }^{5}$ Department of Physics and Astronomy, University of Tennessee, Knoxville, TN 37996

${ }^{6}$ Istituto di Fisica Generale Applicata, University of Milano, I-20133 Milano, Italy

\begin{abstract}
Proton emission half-lives are calculated within the DWBA formalism for ${ }^{131} \mathrm{Eu}$ and ${ }^{141} \mathrm{Ho}$ assuming permanent quadrupole deformation. The decay rates are consistent with a decay from either [411 3/2] or [413 5/2] Nilsson states for ${ }^{131} \mathrm{Eu}$ and [523 7/2] Nilsson state for ${ }^{141} \mathrm{Ho}$.
\end{abstract}

The proton decay of ${ }^{131} \mathrm{Eu}\left(E_{p}=950(8) \mathrm{keV}, t_{1 / 2}=32(9) \mathrm{ms}\right.$ ) and ${ }^{141} \mathrm{Ho}($ $\mathrm{E}_{p}=1169(8) \mathrm{keV}, \mathrm{t}_{1 / 2}=4.2(4) \mathrm{ms}$ ) has been recently reported [1]. The predicted quadrupole deformations for these nuclei are $0.331,0.37$ for ${ }^{131} \mathrm{Eu}$ and $0.29,0.35$ for ${ }^{141} \mathrm{Ho}[2,3]$. Although gamma-ray information for these nuclei has not been reported yet, extrapolation of the first $2^{+}$energy from near-mass measurements is consistent with the predictions These values of $\beta_{2}$, larger than those of the other proton emitters [4], makes these nuclei particularly interesting. It can be argued that since proton emission is sensitive to the potential the proton must tunnel through, which in turn depends on the nuclear shape, it is expected that proton emission will give very valuable information about nuclear shapes.

For proton emitters who have near-spherical shapes, 3 different methods have been developed to calculate the proton emission half-lives (DWBA, WKB and Gurvitz ). A thorough discussion of them has been presented in ref. [5]. So far, only DWBA has been extended to account for axially-symmetric, non-spherical shapes [6]. The same formalism will be used here to calculate half-lives for ${ }^{131} \mathrm{Eu}$ and ${ }^{141} \mathrm{Ho}$.

For a proton moving in a non-spherical potential, the wave function is expanded in terms of spherical wave functions:

$$
\left|\Psi(N \Omega)>=\sum_{j \ell} C_{j \ell}(N \Omega)\right| N \ell j \Omega>
$$




\section{DISCLAIMER}

This report was prepared as an account of work sponsored by an agency of the United States Government. Neither the United States Government nor any agency thereof, nor any of their employees, make any warranty, express or implied, or assumes any legal liability or responsibility for the accuracy, completeness, or usefulness of any information, apparatus, product, or process disclosed, or represents that its use would not infringe privately owned rights. Reference herein to any specific commercial product, process, or service by trade name, trademark, manufacturer, or otherwise does not necessarily constitute or imply its endorsement, recommendation, or favoring by the United States Government or any agency thereof. The views and opinions of authors expressed herein do not necessarily state or reflect those of the United States Government or any agency thereof. 


\section{DISCLAIMER}

Portions of this document may be illegible in electronic image products. Images are produced from the best available original document. 
The coefficients $C_{j \ell}(N \Omega)$ were first obtained by Nilsson using a harmonic oscillator potential. More advanced calculations use Woods-Saxon form factors. The approach followed here is that of ref. [8], the spherical wave functions are obtained numerically ( the Becchetti-Greenlees parametrization [7] is used ), the Hamiltonian matrix is calculated and then diagonalized. The $C_{j \ell}(N \Omega)$ coefficients as well as Nilsson diagrams are calculated this way.

In non-spherical DWBA, the decay amplitude is given by:

$$
\begin{aligned}
B= & \left(\frac{2\left(2 J_{f}+1\right)}{2 J_{i}+1}\right)^{1 / 2}<J_{f} j_{p} 0 K_{i} \mid J_{i} K_{i}>\sum_{\ell j, m_{s}} C_{\ell j}(N \Omega) \times \\
& <\ell_{p} \frac{1}{2}\left(K_{i}-m_{s}\right) m_{s}\left|j_{p} K_{i}><\ell \frac{1}{2}\left(K_{i}-m_{s}\right) m_{s}\right| j K_{i}>\times \\
& <Y_{\ell_{p}}^{K_{i}-m_{s}}\left(\Omega^{\prime}\right) \frac{F_{\ell_{p}}(k, \eta)}{r}\left|V_{D W B A}(\vec{r})\right| \frac{R_{n \ell j}(r)}{r} Y_{\ell}^{K_{i}-m_{s}}\left(\Omega^{\prime}\right)>
\end{aligned}
$$

where $R_{n l j}(r) / r$ is the radial part of the spherical proton wave function, $F_{l}(r)$ is the regular Coulomb function and $V_{D W B A}(r)$ is the total potential minus the monopole Coulomb term.

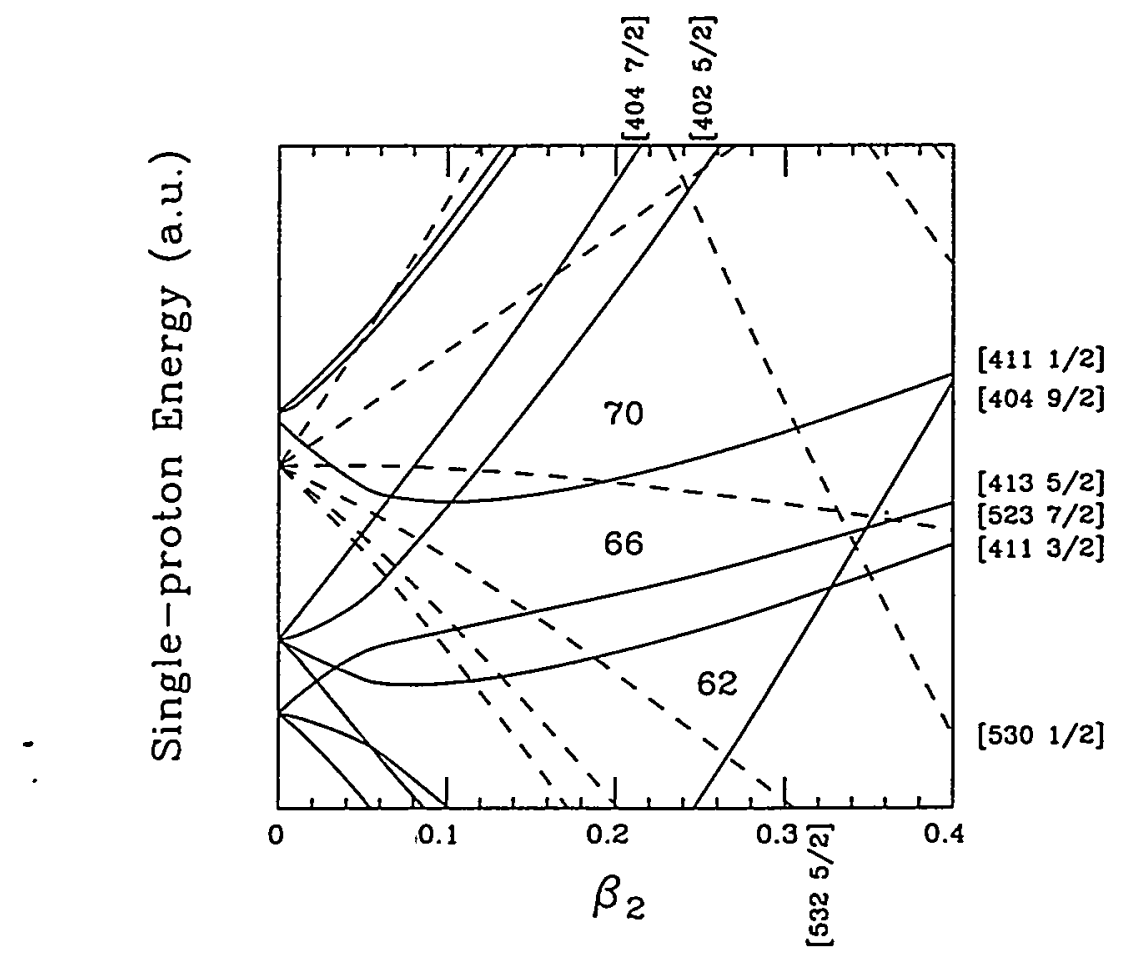

Fig. 1 Proton orbits for nuclei in the vicinity of ${ }^{131} \mathrm{Eu}$ and ${ }^{141} \mathrm{Ho}$ 
The half-life is related to the decay amplitude by:

$$
T_{1 / 2}=\frac{\hbar \ln 2}{2 \pi B^{2}}
$$

As it was for outlined in ref. [6], the observed energy and half-life of the proton decay can be used to determine from which Nilsson orbital the proton originated from. The strategy is to use eq. 2 for a large number of Nilsson orbitals, and presumably, only a few of them will agree with the observations. A Nilsson diagram for protons can be seen in Fig. 1. For quadrupole deformations between 0.2 and 0.4 , the odd proton for $\mathrm{Eu}, \mathrm{Ho}$ and $\mathrm{Tm}$ nuclei will most likely decay from the [411 1/2], [411 3/2], [413 5/2], [402 5/2], [404 7/2] [532 5/2] and [523 7/2] orbitals. We won't consider any nuclear structure effects, i.e. we will take the spectroscopic factor equal to 1. A value of 0.5 was used in ref. [6]. The use of another set of nuclear potential systematics can produce differences by a factor of 2 between calculations. Therefore, we will accept as final candidates those orbitals that are between a factor by 2 to the measured half-lives.

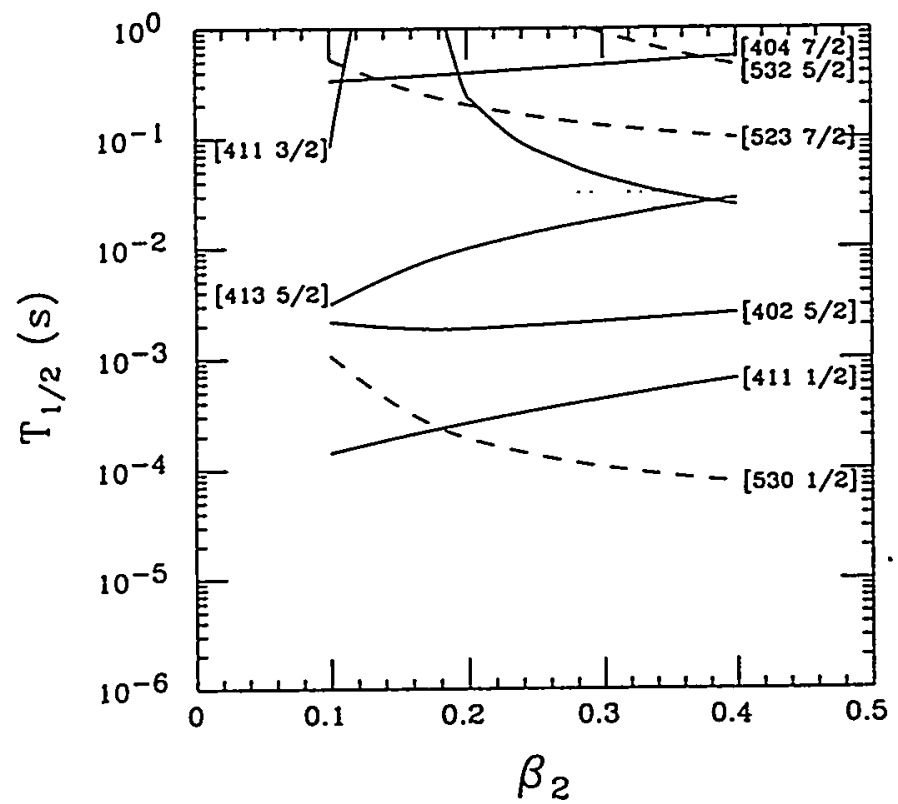
${ }^{131} \mathrm{Eu}$.

Fig. 2 Half-life as a function of deformation for different Nilsson orbits for

The results of the calculations can be seen in figs. 2 and 3 for ${ }^{131} \mathrm{Eu}$ and ${ }^{141} \mathrm{Ho}$ respectively. The measured half-lives are indicated with dotted lines. For ${ }^{131} \mathrm{Eu}$, only calculations from 2 orbitals, [411 3/2] and [413 5/2], match the experimental results at $\beta_{2} \sim 0.35$. For ${ }^{141} \mathrm{Ho}$, the orbitals [523 7/2] at $\beta_{2} \sim 0.3$ and [411 3/2] at $\beta_{2} \sim 0.16$ reproduce the observed results, however only the first is compatible with the expected first excited state of ${ }^{140} \mathrm{Dy}$, likely to be around $175 \mathrm{keV}$. 


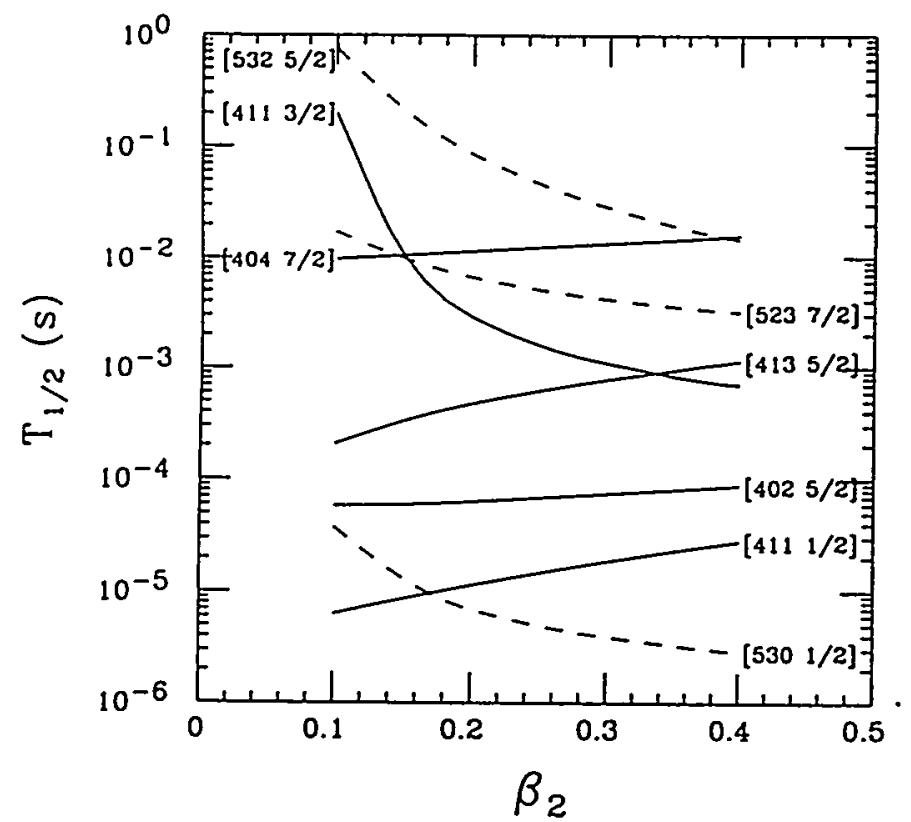

Fig. 3 Half-life as a function of deformation for different Nilsson orbits for ${ }^{141} \mathrm{Ho}$.

In summary, using the measured proton energy and half-life, the Nilsson orbits [411 3/2], [413 5/2] were was assigned to the decay of ${ }^{131} \mathrm{Eu}$ while the [523 7/2] was assigned to ${ }^{141} \mathrm{Ho}$.

\section{References}

[1] C.N. Davids et al, Phys. Rev. Lett. 80, 1849 (1998).

[2] P. Moller et al, At. Data Nucl. Data Tables 59, 185 (1995).

[3] Y. Aboussir et al, At. Data Nucl. Data Tables 61, 127 (1995).

[4] P.J.: Woods and C.N. Davids, Annu. Rev. Nucl. Part. Sci 47, 541 (1997).

[5] S.Aberg, P.B. Semmes and W. Nazarewics, Phys. Rev C56, 1762 (1997).

[6] V.P. Bugrov and S.G. Kadmensky, Sov. J. Nucl. Phys. 52, 229, (1990). S.G. Kadmensky and V.P. Bugrov. Phys. of At. Nuclei 59, 399 (1996).

[7] F.D. Bechetti and G.W. Greenlees, Phys. Rev. 182, 1190 (1969).

[8] B.L. Andersen, B.B. Back, and J.M. Bang, Nucl. Phys. A147, 33 (1970). 